\title{
Generalization of Experimental Information and Identification of Patterns in the Behavior of Metals and Alloys under Fatigue Loading Based on the Mapping of Fatigue Curves into Reduced Spaces
}

\author{
V.V. Andreev ${ }^{1}$, O.V. Andreeva ${ }^{1}$ \\ vyach.andreev@mail.ru|andreevaov@gmail.com \\ ${ }^{1}$ Nizhny Novgorod State Technical University n.a. R.E. Alekseev, Nizhny Novgorod, Russia
}

\begin{abstract}
The paper presents a characteristic of a fairly large sample of data on the fatigue of metals and alloys, including the coordinates of the fatigue limit and a description of the conditions under which these results were obtained. Using the reduction procedure allows us to obtain a generalized dependence of the reduced parameters of fatigue resistance, on the basis of which it is possible to develop forecasting methods. Extension of the reduction procedure to limited endurance limits allows one to obtain a generalized surface of the presented indicators of fatigue resistance and to increase the reliability of the forecasts made of indicators of fatigue resistance.
\end{abstract}

Keywords: metals, fatigue metal, endurance limit, given indicators, reduced dependence, generalized surface, forecasting.

\section{Introduction}

Designing structures, making the right choice of manufacturing and processing methods, ensuring the safe operation of technical devices, extending the life of a device, or making a conclusion about the need for decommissioning when solving all these problems, it is necessary to assess the state of the structural material. The condition of the structural material is determined by its strength indicators, its ability to withstand external loads. In this case, the main type of structural material failure is fatigue. The experimental determination of fatigue resistance indicators is a lengthy and expensive procedure. The desire to reduce the costs of design and production inevitably leads to the need to predict the properties of metals and alloys based on previously obtained experimental data, combining them as part of some integral models for describing the properties of structural materials under unsteady loading.

Despite its high cost, the numerous data on fatigue of metals and alloys obtained by different authors represent a poorly structured, multidimensional array of information. The study of such data, for the purpose of generalization, is difficult. Quite often it is impossible to combine and jointly review the results of individual studies. A large amount of data remains unknown to a specific specialist, and it is often impossible to use this information effectively due to the publication of limited information. Quite often, there remains only the possibility of an almost intuitive assessment of the influence of a particular factor from the totality of acting factors that only an expert can fulfill.

Most of the empirical dependencies proposed in the literature, which relate the values of the sought-for indicators of metal fatigue resistance to various calculated or experimentally obtained parameters, have a limited range of definitions and are difficult to reconcile when trying to jointly account within a single information system.

Thus, the need to develop a procedure for converting experimental data on the fatigue of metals and alloys, which allows combining available poorly structured, heterogeneous information, is obvious. It is necessary, using the analysis of accumulated experimental data and existing methods of limited generalization of information for individual groups of metals and alloys within separate sets of factors, to try to develop a universal system for generalizing information on the fatigue of metal materials and to synthesize a model that describes the behavior of metals and alloys under cyclic loading as a single class construction materials.

\section{Materials and method}

There are various forms of graphical representation of experimental metal fatigue data. The most convenient option is the representation of the fatigue curve in a system of logarithmic coordinates. In this case (under certain experimental conditions), the multi-cycle region and the endurance region are represented by two segments of straight lines intersecting at the fracture point. The angle of inclination of the fatigue curve to the axis of the number of loading cycles is the so-called structurally sensitive parameter, an indicator of the intensity of damage accumulation processes in a structural material under cyclic loading. In fig. 1 is a schematic representation of a multi-cycle region of a fatigue curve in a logarithmic coordinate system.

The special nature and causes of the appearance of a fatigue curve fracture in a logarithmic coordinate system provide an opportunity for the implementation of various forecasting methods. From the point of view of stability theory, the inflection point on the fatigue curve is a bifurcation point, the appearance of which indicates a fundamentally different behavior of the structural material in the right horizontal section, compared with the left section of the fatigue curve that is steeply inclined to the axis of the number of loading cycles.

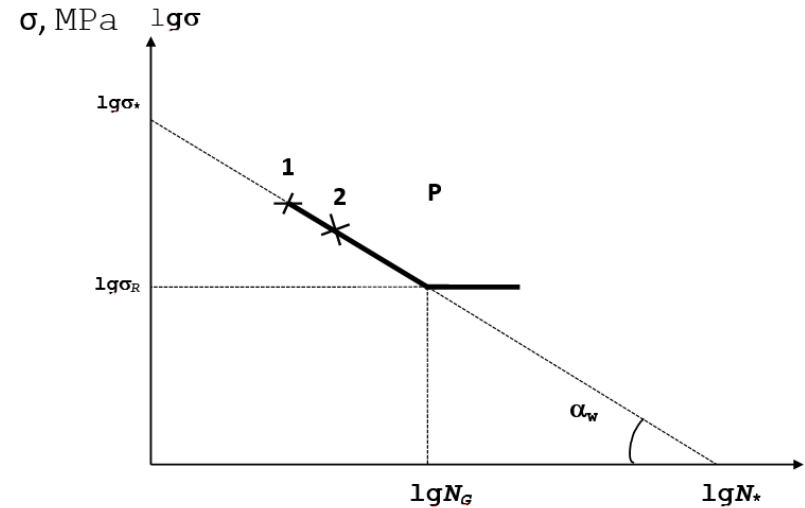

Fig. 1. Schematic representation of the multi-cycle region of the fatigue curve in a logarithmic coordinate system: $P$ fatigue limit (point of fracture of the fatigue curve); $N$ - is the number of loading cycles; $\sigma_{\mathrm{R}}$ is the stress $(M P a)$ corresponding to the endurance limit; $N_{\mathrm{G}}$ is the abscissa of the point of fracture

of the fatigue curve (the number of loading cycles

corresponding to the transition of the fatigue curve to a horizontal section); $\alpha_{\mathrm{w}}$ is a structurally sensitive parameter of metal fatigue resistance; $\sigma_{*}, N_{*}$ are conditional, physically

unrealizable values of stress and durability at which a straightened fatigue curve intersects the coordinate axes, 1 and 2 are experimental points corresponding to the destruction of

the objects under study after a certain number of loading cycles $N_{1}$ and $N_{2}$ at given stressed levels $\sigma_{1}, \sigma_{2} ; \lg \sigma=\lg \sigma_{*}-$ $\operatorname{tg}\left(\alpha_{W}\right) \lg _{N}$ equation of the left branch of the fatigue curve.

For joint consideration, more than 1000 fatigue curves of metals and alloys were selected during the construction of which 
the endurance limits were experimentally determined. These endurance limits (the intersection points of the left and right sections of the fatigue curve in the multi-cycle region) are presented in fig. 2. Table 1 shows the results of the systematization of the selected experimental data. This systematization made it possible to determine the "dimension" of the feature space characterizing the data selected for analysis. These signs are a description of the factors (conditions) under which periodic loading of laboratory samples and field parts was carried out.

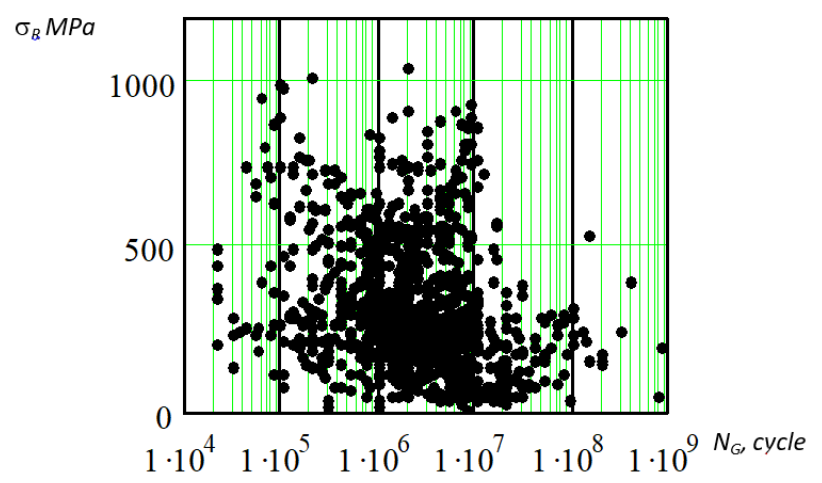

Fig. 2. The position of the fracture points of the metal fatigue curves in the multi-cycle region considered in the study ( $\sigma_{\mathrm{R}}$ is the stress, $\mathrm{MPa} ; N_{\mathrm{G}}$ is the number of loading cycles)

Table 1 shows the results of the systematization of the selected experimental data. This systematization made it possible to determine the "dimension" of the feature space characterizing the data selected for analysis. These signs are a description of the factors (conditions) under which periodic loading of laboratory samples and field parts was carried out.

Table 1

A generalized description of factors under the action of which the results of fatigue tests of laboratory samples and field parts considered in the study were obtained

\begin{tabular}{|c|c|c|}
\hline № & Name of factor & $\begin{array}{c}\text { Range of variation } \\
\text { (number of different } \\
\text { options, levels, methods, } \\
\text { modes) }\end{array}$ \\
\hline 1. & Steel or alloy grade & 204 \\
\hline 2. & $\begin{array}{c}\text { Method of loading } \\
\text { (scheme) }\end{array}$ & 17 \\
\hline 3. & Test environment & 54 \\
\hline 4. & Test temperature & 66 (from -269 to $1000^{\circ} \mathrm{C}$ ) \\
\hline 5. & Cross sectional shape & 4 \\
\hline 6. & $\begin{array}{c}\text { Scale factor } \\
\text { (characteristic size) }\end{array}$ & 65 (from 1 to $111 \mathrm{~mm}$ ) \\
\hline 7. & Heat treatment mode & 81 \\
\hline 8. & $\begin{array}{c}\text { Surface treatment } \\
\text { method }\end{array}$ & 28 \\
\hline 9. & Surface finish & 63 (from $\mathrm{Ra} 0,2$ to $\mathrm{Ra}$ \\
\hline 10. & Cycle frequency & 68 (from 2,5 to $20000 \mathrm{~Hz}$ ) \\
\hline
\end{tabular}

A known problem arises when it is necessary to compare two different fatigue curves. Taking into account only the strength characteristics (for example, the endurance limit value or the values of the limited endurance limit) or the durability characteristics (for example, comparing the abscissa of the inflection points of two fatigue curves) or even comparing the values of structurally sensitive parameters (the slope angles of the compared fatigue curves) does not allow get an unambiguous answer about the advantage of one or another of the considered options for the location of the fatigue curve. Some complex indicators of fatigue resistance are needed, which would take into account indicators of both strength and durability, and the stability of the structure under cyclic loading.

\section{Literature review}

A huge number of scientific papers have been devoted to the study of the phenomenon of fatigue both in our country and abroad. Since the moment of the "conscious" study of the phenomenon of fatigue, which is associated with the studies of the German engineer Weller (1858), over 30,000 scientific papers have been published on this problem. Among the scientists who made a significant contribution to the study of the phenomenon of fatigue, I.A. Odinga, V.T. Troshchenko, V.S. Ivanov, V.P. Kogaev, V.M. Grebennik, I.V. Kudryavtseva, V.F. Terentyeva L.M. Akimova, N.V. Oleinika, Griffith A.A., Orowan E., Coffin L.F., Mott N.F., Cottrel A.H., Yokobori T. and others [3-5, 7-10]. The development of methods for accelerated determination of fatigue resistance indicators was the subject of research by Stepnova M.N., Troshchenko V.T., Evstratova S.P., Panteleeva V.N., Goltseva D.I., Ivanova V.S. and Gordienko L.K., Yatskevich S.I., Muratova L.V. [6] I.A. was engaged in a quantitative analysis of the influence of various factors on indicators of fatigue resistance and the development of a system for accounting for their joint action. Oding, N.I. Kononchuk, L.M. Akimov, D.I. Shetulov, V.P. Kogaev and others $[1,6,7]$

Long-term studies of the phenomenon of fatigue made it possible to accumulate significant amounts of information on the experimental evaluation of the results of periodic effects of various kinds on structural material, however, as noted in the literature, researchers have not brought to the final solution to the problem of accurately predicting the results of fracture processes in structural materials when this kind of operational impact. There is no complete understanding of the physical nature of fatigue; there is no way to accurately determine the time of onset of failure. It is customary to talk about established patterns or hypotheses of fatigue failure, implemented using various, for example, energy, dislocation, statistical, or other scientific theories to explain the processes of material failure under cyclic loading.

The variety of accumulated experimental data confirms the complexity of the behavior of metals and alloys during fatigue, does not allow us to unequivocally accept, as the only one that corresponds to the observed results, none of these hypotheses.

At the same time, starting from the 70s of the last century, among scientists (see, for example, the works of Ivanova V.S. and Terentyev V.F. [7]), the belief that further practically useful results in this field spread possible only on the basis of interdisciplinary research, including with the involvement of the methodology of synergetics, information processing theory and system analysis [9]. The basis for such conclusions was the work of Prigozhin I., Stengers I., Haken G., Stanley H., Klein U., which made it possible to justify the consideration of metal as a complex dynamic system located at the time of destruction far from the equilibrium state and to apply the methodology for analyzing the stability of dynamic systems, the theory of catastrophes, to more thoroughly study the jump-like transitions of systems from one state to another (bifurcation points).

Despite its objectively high cost, the numerous data obtained by various authors on the fatigue of metals and alloys are a poorly structured, multidimensional array of information, the study of which, and the development of any recommendations based on it, are difficult. The substantial part of the results of fatigue tests is often due to the narrow tasks of a specific study. This leads to the impossibility of combining and jointly considering the results of individual studies, and obtaining, on the basis of their analysis, practically useful generalized information. A large amount of 
data remains unknown to a specific specialist, and it is often impossible to use this information effectively due to the publication of limited information. Quite often, there remains only the possibility of an almost intuitive assessment of the influence of a particular factor from the totality of acting factors that only an expert expert can perform.

\section{Development the space of reduced parameters}

To obtain preliminary experimental data on the fatigue of metals and alloys. In order to obtain relative parameters (given indicators of fatigue resistance), in the coordinates of each point belonging to the left branch of the stress curve, refer to the conditional values of the stress and the number of loading cycles in which the conditionally continued fatigue curve intersects the coordinate axes:

$$
\begin{gathered}
\sigma_{\text {reduced }}=-\log \left(\sigma_{R} / \sigma_{*}\right), \\
N_{\text {reduced }}=-\log \left(N_{G} / N_{*}\right), \\
\operatorname{tg} \alpha_{W_{\text {reduced }}}=-\log \left(\operatorname{tg} \alpha_{W}\right),
\end{gathered}
$$

The proposed transformation allows you to translate any fatigue curve from a system of logarithmic coordinates to one surface in the three-dimensional space of the transformed coordinates. Moreover, the generatrix of this surface is a curve of the form $y=(\log (x))-A$, and the guide is parallel to the axis of the transformed angle of inclination of the fatigue curve.

A schematic image of the surface under consideration is presented in fig. 3 .

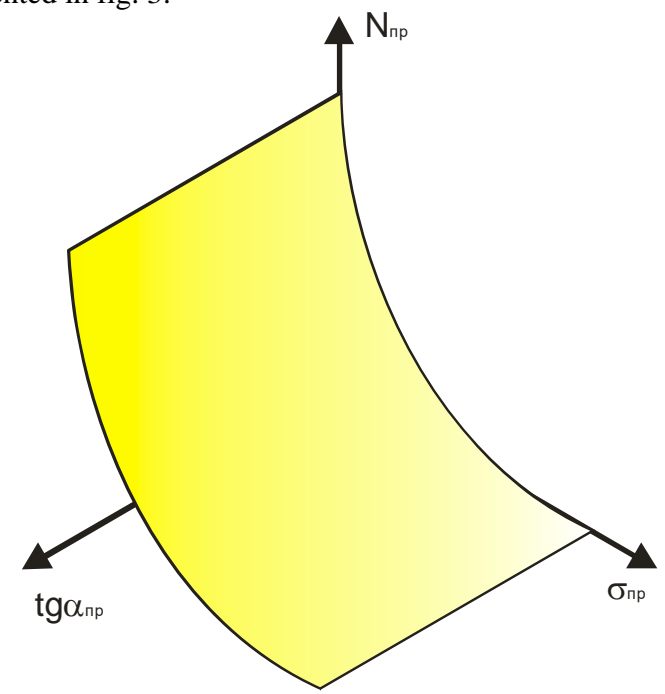

Fig. 3. Schematic representation of the surface within which the normalized space of fatigue curves is stratified by an informative parameter

In [4], on the basis of processing the obtained dependences, the following approximation expressions for the projections of the generalized dependence were obtained:

$$
\begin{gathered}
\sigma_{\text {reduced }}=-\log \left(\sigma_{R} / \sigma_{*}\right), \\
N_{\text {reduced }}=-\log \left(N_{G} / N_{*}\right), \\
\operatorname{tg} \alpha_{W_{\text {reduced }}}=-\log \left(\operatorname{tg} \alpha_{W}\right),
\end{gathered}
$$

The confidence coefficient for dependencies (4-6) was not lower than 0.93 .

Given the large number of points (fatigue curves) considered to obtain these dependences, we can talk about some generalized dependences of the behavior of a metal structural material under the action of a cyclic load under different sets of factors.

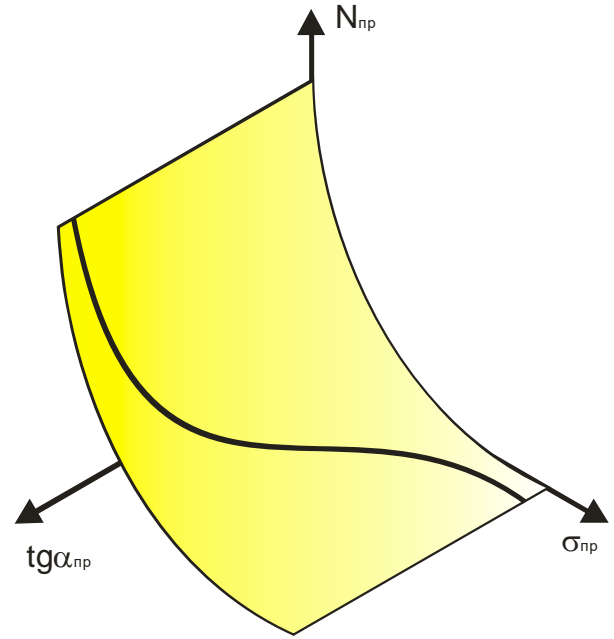

Fig. 4. Schematic representation of the surface of the given parameters of fatigue resistance with points highlighted on it, corresponding to the physical endurance limits of metals and alloys

Consideration of all presented in fig. 1 experimental fatigue curves in the space of reduced coordinates allows you to get the following picture (fig. 5, 6).

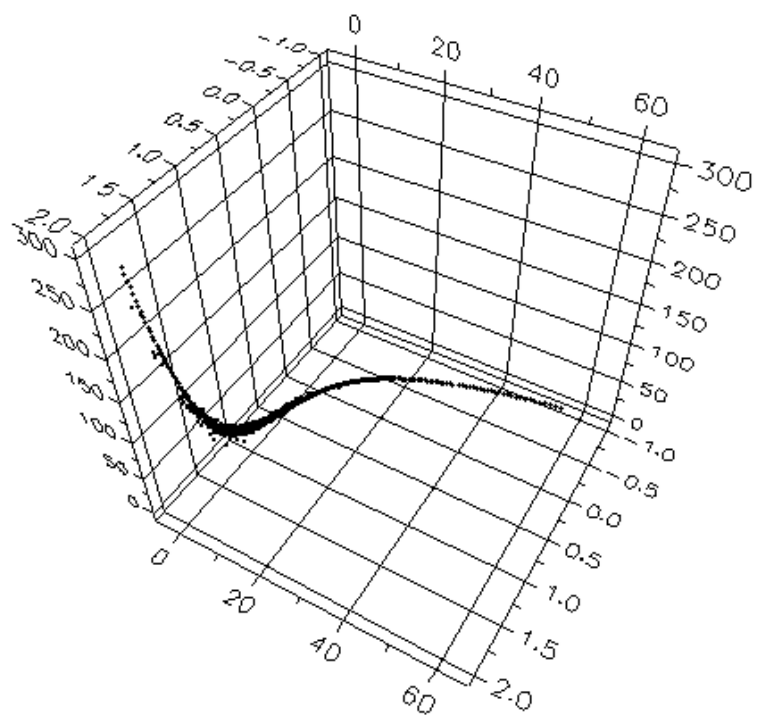

Fig. 5. Generalized dependence of the given parameters of fatigue resistance of metallic materials

In other words, we got a new object of study - a transformed analogue of the studied subject area - an image of fatigue curves in space of the given indicators of fatigue resistance.

We repeat that the generalized dependence was obtained while considering the endurance limits obtained experimentally on various fatigue curves. Comparing with each other the points on the fatigue curves, which are the same number of times apart from the endurance limits, we can obtain dependences similar to the generalized dependence of the reduced parameters of fatigue resistance in terms of universality.

An examination of the family of such curves makes it possible to obtain a generalized surface of the reduced parameters of fatigue resistance. The visualization program for the generalized surface of the reduced parameters allows not only to study the generalized dependence from different observation points, but also to accurately position the arbitrary fatigue curve in the space of reduced coordinates by setting the angle of inclination of the fatigue curve and the stressed level. 


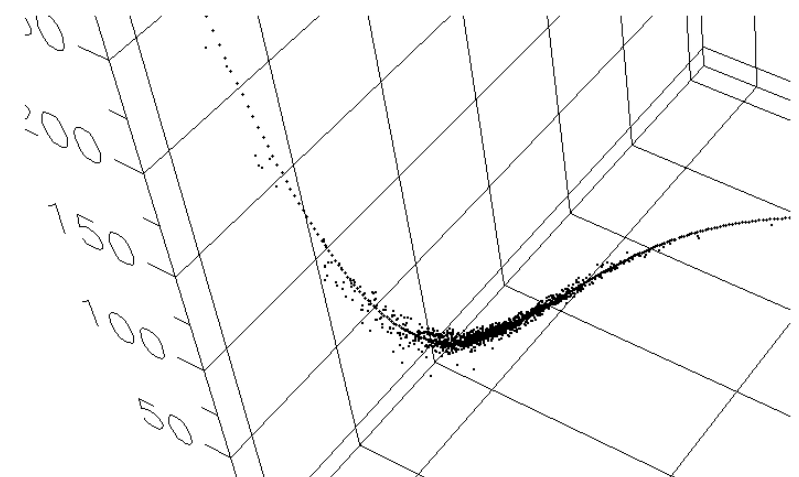

Fig. 6. The enlarged part of the generalized dependence of the given parameters, containing the bulk of the experimental points

Expressions were obtained for the projections of the lines corresponding to the same values of the stressed level (the difference between the current stressed value and the value of the physical endurance limit):

$$
\begin{aligned}
& N_{\text {reduced }}=C * \exp \left(D * \operatorname{tg} \alpha_{W_{\text {reduced }}}\right), \\
& \sigma_{\text {reduced }}=A * \exp \left(B * \operatorname{tg} \alpha_{W_{\text {reduced }}}\right),
\end{aligned}
$$

the coefficients A, B, C and D in which are related to the coefficient $\mathrm{k}$, which shows how many times the current value of the endurance limit exceeds the physical endurance limit, in accordance with the following expressions:

$$
\begin{gathered}
\mathrm{C}=0,4416 * \ln (k)+1,9383, \\
D=-0,0531 * \ln (k)+2,5247, \\
A=6,1287 * k^{2}-5,5584 * \mathrm{k}+5,868, \\
B=-0,7332 * k^{2}-0,4453 * \mathrm{k}-1,1474 .
\end{gathered}
$$

The last two expressions are applicable when $0.3<=k<=$ 1.15. If a priori it is assumed that the limited endurance limit exceeds the physical endurance limit by more than $15 \%$, instead of calculating the coefficients $\mathrm{A}$ and $\mathrm{B}$, you must use the expression:

$$
\begin{gathered}
\sigma_{\text {reduced }}=2,2499 * \operatorname{tg} \alpha_{W_{\text {reduced }}{ }^{4}-10,831 * \operatorname{tg} \alpha_{W_{\text {reduced }}}{ }^{3}+} \\
19,903 * \operatorname{tg} \alpha_{W_{\text {reduced }}{ }^{2}}-\operatorname{tg} \alpha_{W_{\text {reduced }}}+F, \\
F=0,0455 * k^{2}-0,4222 * \mathrm{k}+7,3732 .
\end{gathered}
$$

For the coefficients C and D (in the case of calculating the dependence for $\mathrm{Npr}$ ), the calculated expressions do not change even if the coefficient $\mathrm{k}$ falls outside the range indicated above.

In fig. 7 shows the main elements of the space of reduced fatigue resistance indices: experimental points corresponding to the physical endurance limits of fatigue curves obtained experimentally, a generalized dependence of the reduced fatigue resistance indices, and a generalized surface of the reduced fatigue resistance indices whose equal level lines correspond to the same stressed value, i.e., the same number of times differs from the value of the physical endurance limit.

The study of the results of the parallel representation of fatigue curves in the traditional and reduced coordinate systems allowed us to confirm the greater convenience of the reduced coordinate system for quantifying the effect of various factors on the fatigue resistance parameters. In particular, the dependences of the parameters of the generalized dependence were obtained for various combinations of acting factors.

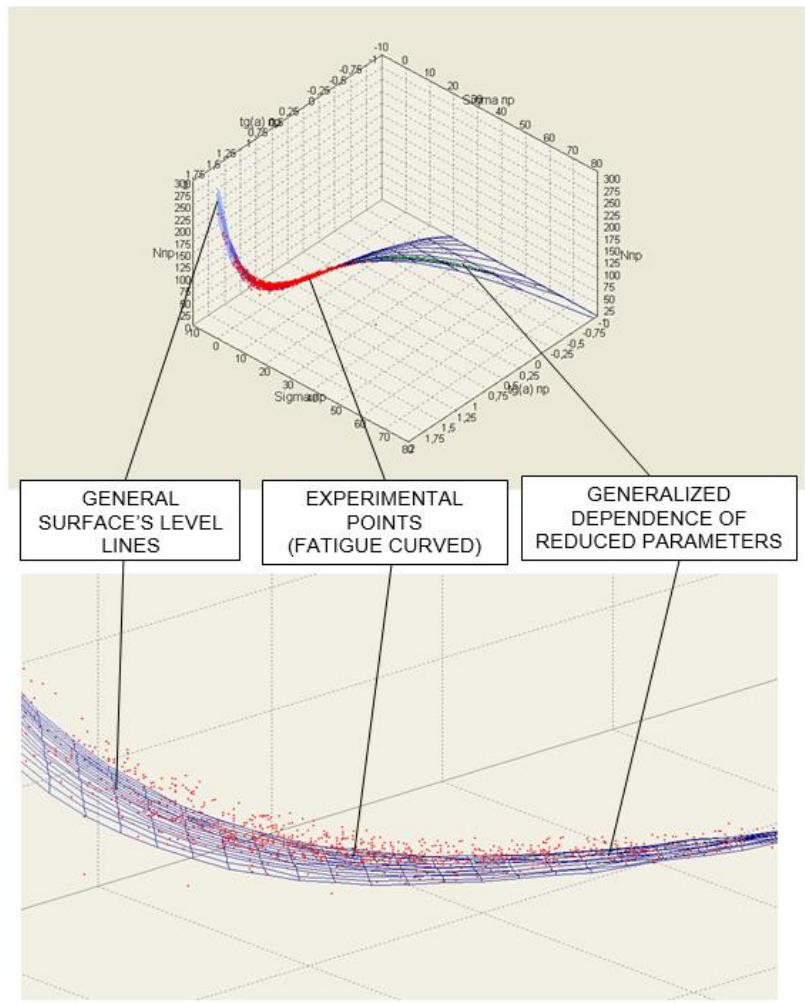

Fig. 7. The main elements of the space of reduced indicators of fatigue resistance of metals

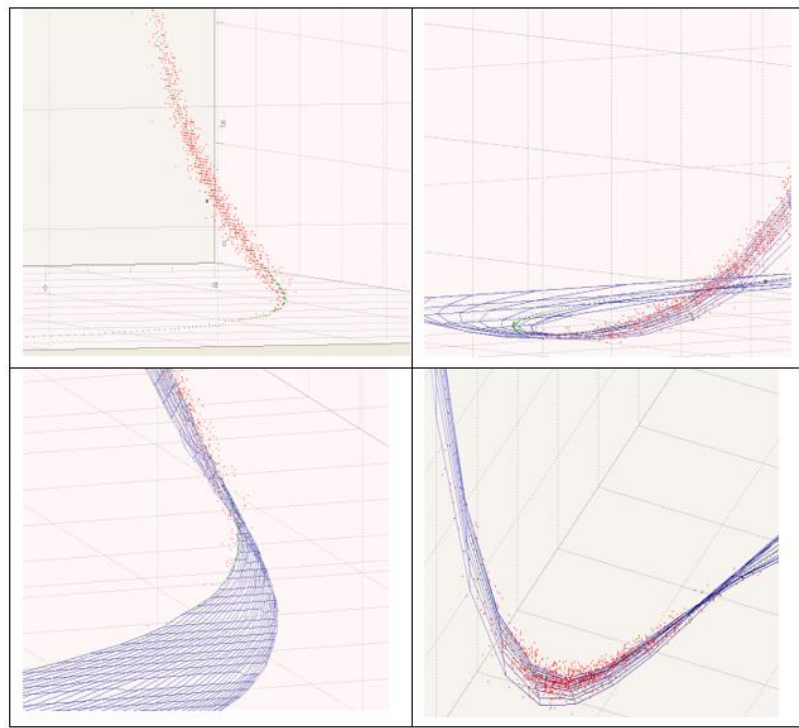

Fig. 8. View of the generalized dependence, the generalized surface of the reduced parameters of fatigue resistance and experimental points corresponding to the region of small angles of inclination of the fatigue curves to the axis of the number of loading cycles

\section{Results}

Using the above indicators of fatigue resistance, it was possible to develop a method for joint consideration of experimental data on the fatigue of metals and alloys. As a result of this, a generalized dependence of the reduced parameters of fatigue resistance was obtained. The domain of determination of the obtained generalized dependence and the method for predicting fatigue resistance indicators based on the use of this generalized dependence are estimated. The forecasting method is 
quite universal, it is applicable to describe the behavior of a wide range of steel and alloy grades under various combinations of acting factors. At the same time, this method has a limitation - it is applicable only under the condition when the existence of a fracture point (physical endurance limit) is assumed in the multicycle region of the fatigue curve.

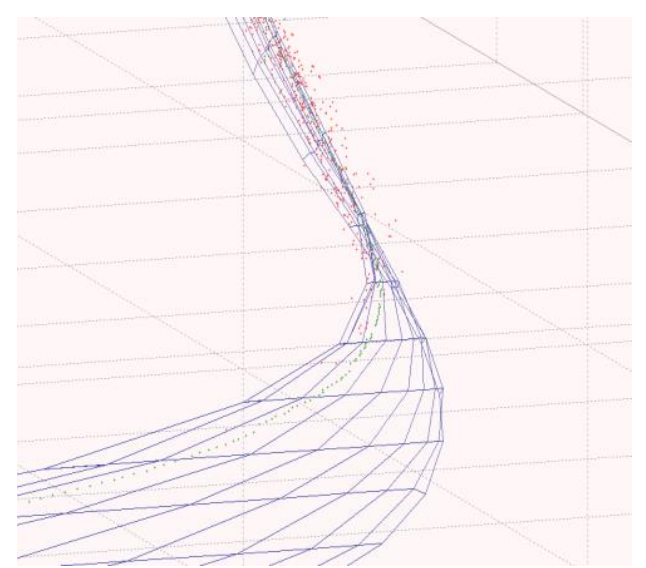

Fig. 9. Elements of the space of reduced coordinates near the transition to large angles of inclination of the fatigue curve to the axis of the number of loading cycles. The generalized dependence of the reduced parameters of fatigue resistance is represented by a consecutive series of points. Added lines of equal voltage level (make up a system of isolines uniform with the generalized dependence) and grid lines of the generalized surface corresponding to equal values of the reduced angle of inclination of fatigue curves (for the presented case, the step of changing the reduced angle of inclination when constructing the generalized surface is 0.2 ; when constructing the generalized dependence, the step less; the ratio of stress to the endurance

limit varies from 0.3 to 1.3 in increments of 0.1 . The

generalized relationship corresponds to a ratio of 1 ).

Comparison with each other of points of different fatigue curves that differ from endurance limits by the same number of times made it possible to construct a generalized surface of the reduced parameters of fatigue resistance. Using the generalized surface of the given parameters of fatigue resistance made it possible to compare and generalize, within the framework of a single, practically functional dependence, the data on fatigue tests of metals and alloys in the case when the tests were carried out at a base less than the abscissa of the inflection point of the fatigue curve.

\section{Gratitudes}

The work was supported by RFBR, Grant № 19-07-00455.

\section{References}

[1] Andreev V.V. The endurance limit of metals on the generalized dependence of the given parameters of fatigue resistance.- N.Novgorod: Nizhny Novgorod. Univ., 2003.

[2] Andreeva O.V. Model and algorithms for assessing the damage to the surface microstructure of metals and alloys from images / Monograph. NSTU named after R.E. Alekseeva, N. Novgorod, 2018, 5 ps.

[3] Ekobori T. Physics and mechanics of fracture and solid strength. M.: Metallurgy, 1971. - 264 p.

[4] Ivanova V.S. Fatigue failure of metals. - M. Metallurgizdat, 1963.- 272 p.

[5] Kogaev V.P. Some questions of the fatigue strength of steel.-M .:, 1953.- P.126-132.

[6] Oleinik N.V., Sklyar S.P. Accelerated fatigue tests. - Kiev: Science. Dumka, 1985.- 304 p.
[7] Terentyev V.F. Fatigue of metallic materials. -M .: Nauka, 2003. -- $248 \mathrm{p}$.

[8] Terentyev V.F. Fatigue strength of metals and alloys. M .: Intermet Engineering. - 2002. - 287 p.

[9] Troshchenko V.T. Deformation and fracture of metals under multi-cycle loading. - Kiev: Science. Dumka, 1981.- 343 p.

[10] Troshchenko V.T., Sosnovsky L.A. Fatigue resistance of metals and alloys. - Kiev: Science. Dumka, 1987.- 1303 p. 